\title{
The Application of High-speed Videogrammetric Technique in Underwater 3D Displacement Measurement Scenario
}

\author{
Peng Chen ${ }^{1,2}$, Jiajun Zhao ${ }^{1+}$, Sa Gao ${ }^{1}$, Benkang Wang ${ }^{1}$, Huangping Ma ${ }^{1}$ and Xiaohua Tong ${ }^{1,2}$ \\ ${ }^{1}$ College of Surveying and Geo-Informatics, Tongji University, Shanghai, 200092, China \\ ${ }^{2}$ State Key Laboratory of Disaster Reduction in Civil Engineering, Tongji University, Shanghai 200092, \\ China
}

\begin{abstract}
With the rapid development of underwater engineering, acquiring accurate three-dimension (3D) measurement of underwater objects has become a very concerned topic. High-speed videogrammetry, which has advantages of non-contact measurement, flexibility and high precision, provides a feasible method to extract transient 3D displacement of objects. This paper presents the application of high-speed videogrammetric technique in underwater 3D displacement measurement scenario, which includes the following four main components: (1) An underwater high-speed videogrammetry network is established to capture and record the 3D displacement of underwater objects; (2) The underwater camera calibration is performed to obtain the interior orientation parameters and eliminate lens distortion; (3) Accurate target recognition and tracking algorithms are introduced to calculate the image coordinates of the target points in the high-speed image sequence; (4) 3D displacement is calculated based on 3D reconstruction of target points on objects via videogrammetric analysis algorithms. The underwater simulation experiment indicates that the proposed technique has a fairly good application performance in underwater measurement scenario with submillimeter accuracy
\end{abstract}

Keywords: High-speed videogrammetry, Underwater measurement, Image sequence processing, 3D displacement measurement

\section{Introduction}

With more and more large-scale development and utilization activities in underwater engineering, precise 3D measurement of underwater structures is currently a concerned research issue in photogrammetry community [1-2]. Taking the underwater structural health monitoring experiment as an instance, the seismic scenario is simulated by an underwater shaking table, and the displacement process of the structure in the underwater environment is measured by encountering different levels of seismic actions, thereby verifying the feasibility of structure designs. In simulated test experiments, in order to explore the ultimate loading capacity of the structure and the displacement caused by the structure-water coupling interaction, it is crucial to constantly measure the damage and displacement of the structure [3-4].

Traditional engineering measurement solutions are mainly based on contact sensors, including displacement gauges, accelerometers, strain gauges, etc., which are usually installed on the surface of the object to measure the movement of the experimental object. However, these measurement methods are clumsy for displacement monitoring of underwater structures due to the limited measurement range, small measurement area, difficulty in assembly, increased model weight and platform stability dependence [5-7]. High-speed videogrammetry, as a non-contact and uncomplicated optical measurement method, can accurately record the transient movement state of objects in detail, and then calculate the 3D displacement of the objects through the photogrammetric analysis algorithm [8].

\footnotetext{
+ Corresponding author. Tel.: + 8618930520221.

E-mail address: tjubbd@163.com.
} 


\section{Methodology}

The underwater high-speed videogrammetry scheme, as shown in Fig. 1, includes the following four main parts: (1) Underwater high-speed videogrammetry network; (2) Underwater camera calibration; (3) Target recognition and tracking; (4) Three-dimension reconstruction.

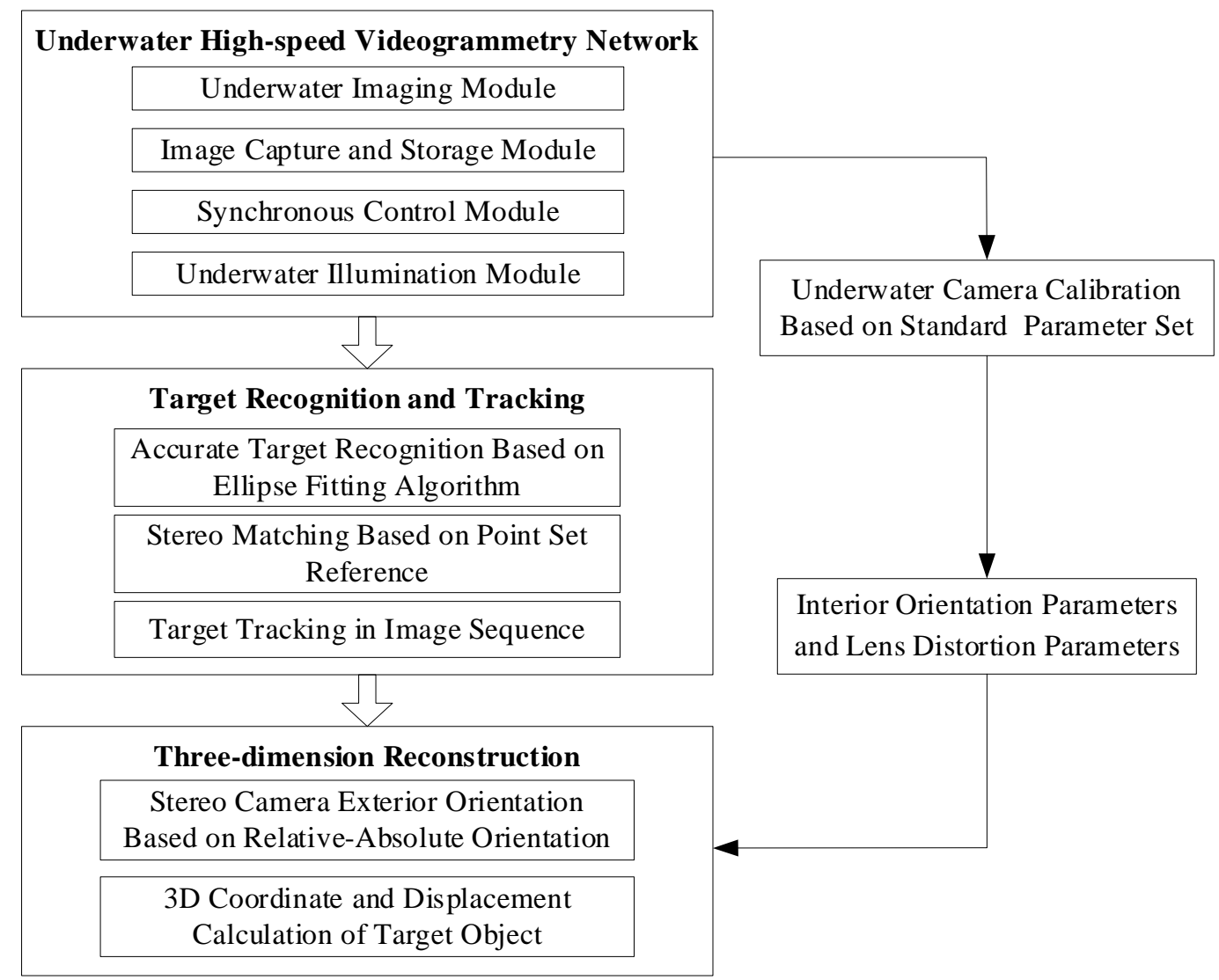

Fig. 1: Flowchart of underwater high-speed videogrammetry scheme.

\subsection{Underwater High-speed Videogrammetry Network Construction}

The underwater high-speed videogrammetry network consists of four parts: (1) Underwater imaging module: High-speed industrial camera, which is the main hardware to record the morphological changes of fast-moving objects in detail. Also, the cameras are specially under waterproof treatments to meet the needs of underwater observation; (2) Image capture and storage module: The main function of this module is to capture and store huge numbers of image sequences from high-speed cameras in real-time; (3) Synchronous control module: It plays an important role in making high-speed cameras capture image sequences simultaneously in the same camera network, which is the key prerequisite for 3D reconstruction in highspeed videogrammetry; (4) Underwater illumination module: Out of the consideration of underwater light supplement and water resistance, waterproof LED lights are selected as illumination equipment, though the natural light is the best choice because of its uniform lighting.

\subsection{Underwater Camera Calibration}

Underwater camera calibration aims to obtain the interior orientation parameters and lens distortion parameters of the camera under water. This paper chooses camera calibration method based on the standard parameter set, which is the most flexible and convenient method [9]. The theory of this method is to directly apply the traditional camera calibration algorithm to the underwater camera calibration, with the refraction geometry being forced to be regarded as the optical straight line geometry, and the error generated will be evenly apportioned to both interior orientation parameters and lens distortion parameters of the camera. When the main optical axis of the camera is perpendicular to the surface of the medium, the refraction distortion error caused by light refraction can be absorbed by the lens radial and tangential distortion parameters. This method can obtain quite high calibration accuracy in the field of view during underwater 
measurement. For the sake of waterproof, the checkerboard pattern is printed on a flat steel plate, as shown in Fig. 2.



Fig. 2: Steel checkerboard (Board size: 10×7; Square size: 50mm).

\subsection{Target Recognition and Tracking}

The scattering effect under water will cause the light to be unevenly distributed on the surface of the object, which has a serious impact on target recognition and tracking, and even result in misrecognition or mismatch. Circular artificial signs are hence used to mark the target points to improve the positioning accuracy of the measurement target points. To accurately extract the centre of the circular artificial sign, least-squares ellipse fitting method is used due to its centre positioning accuracy being hardly affected by images of non-uniform gray value [10]. Circular artificial signs and corresponding ellipse fitting effect are shown as Fig. 3.

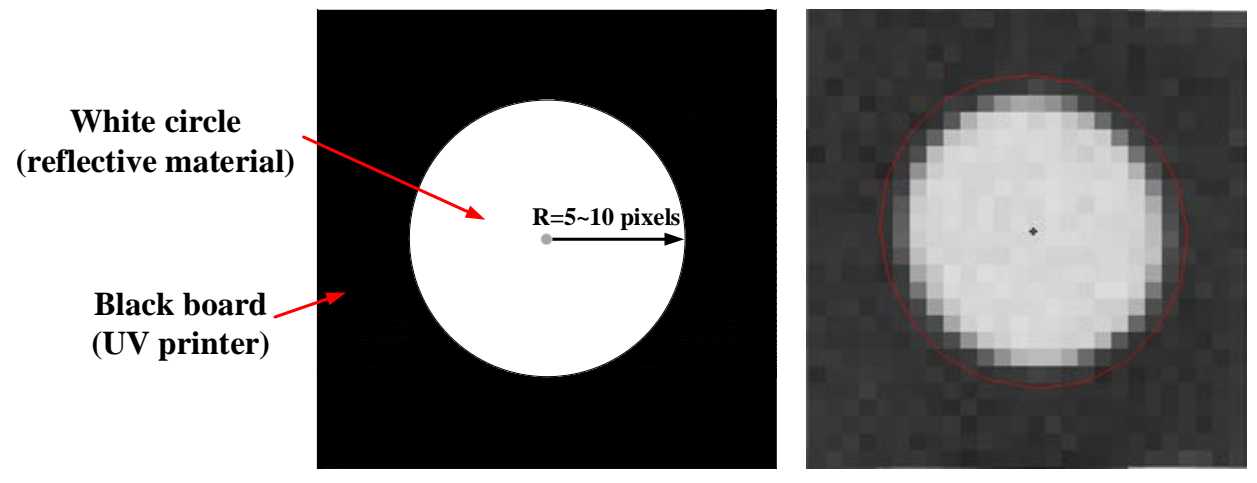

Fig. 3: Circular artificial signs and ellipse fitting result.

After target recognition, stereo matching task is completed by point set reference. During target tracking period, a coarse-to-fine matching strategy is applied to image sequences. Given the initial target position in Frame $i$ along with the determined track window radius, the coarse matching process is carried out based on zero-mean normalized cross-correlation (ZNCC) criteria [11], which evaluates the similarity between the initial target pixel block in Frame $i$ and the pixel block to be matched in Frame $i+1$. After that, least squares matching (LSM) is used to calculate the precise tracked position of the target in Frame i+1, which is namely the fine matching process [12]. As shown in Fig. 4, the detailed target matching and tracking process is illustrated. In the experiment of this paper, the track window radius is set to 10 pixels since the circular signs' radii are among $5 \sim 10$ pixels.



Fig. 4: Target matching and tracking in image sequence processing. 


\subsection{D Reconstruction}

Considering the inconvenience of underwater experiment scenario and thus few control points laid in the common field of view of stereo cameras, relative-absolute orientation is used to estimate stereo camera exterior orientation and 3D reconstruct the target point sequence with space forward intersection algorithm. Relative orientation refers to restoring or determining the relationship between conjugate rays, that is, to solve the relative orientation elements of the stereo image pair. Then the point projection coefficient method can be used to solve the 3D coordinates of all target points (or tracking points) in each frame in the world coordinate system with the given common target points and control points whose 3D world coordinates are measured by total station in advance. The overall 3D reconstruction process is illustrated as Fig. 5.



Fig. 5: 3D reconstruction process based on relative-absolute orientation.

\section{Experiment and Result}

\subsection{Experiment Background and Details}

The purpose of this experiment is to verify the high-speed videogrammetric technique's capability of measuring objects' 3D displacement underwater. An artificial multi-story structure made of steel pipes serves as the measurement object, on which some circular signs are stuck as target points and control points, as shown in Fig. 6(a). Also, some control points are stuck on pipes outside the structure plane to establish 3D control field. The stereo cameras are fixed on a rigid frame fixed on the pool wall as shown in Fig. 6(b). The resolution of the image is $2304 \times 1702$ pixels, and the frame rate was set as $150 \mathrm{fps}$. Each camera is equipped with a $20 \mathrm{~mm}$ fixed focus lens to increase the field of view. Moreover, the 3D coordinates of the control points are measured by a Sokkia NET05AX total station of $0.5 \mathrm{~mm}$ accuracy to provide a reference world coordinate system and also to verify the accuracy of videogrammetric 3D reconstruction algorithm.

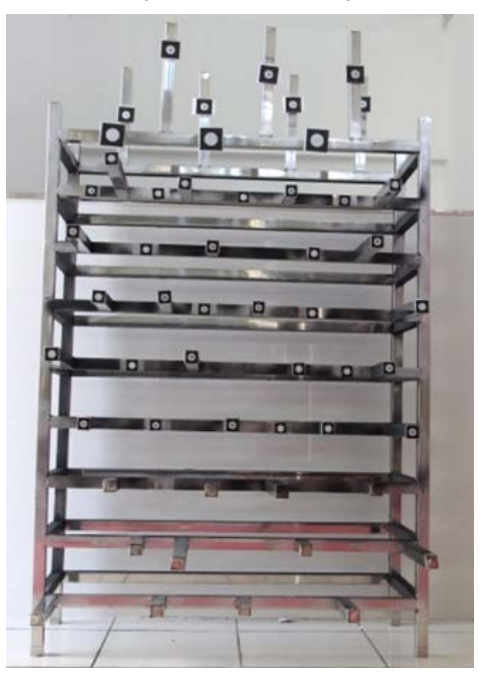
stereo cameras layout; (c) Experimental observation scene. 
3D displacement will happen to the structure during the wave-making process by equipment, and then the high-speed videogrammetric technique is applied to record and measure the 3D displacement of the underwater structure.

\subsection{Results and Analysis}

The underwater camera calibration result indicates that the average back projection errors of the highspeed cameras in image plane are among $0.05-0.3$ pixels, which is a satisfactory calibration result. To verify the accuracy of videogrammetric 3D reconstruction algorithm, some control points are selected as check points and their 3D coordinates are interpolated with the external orientation parameters calculated by other control points. Then the interpolated 3D coordinates and the ones measured by total station of the check points are compared and the discrepance is shown as Table 1 . The location accuracy $\left(\sqrt{\sigma_{X}^{2}+\sigma_{Y}^{2}+\sigma_{Z}^{2}}\right)$ of the point in high-speed videogrammetric scheme can reach around $0.65 \mathrm{~mm}$, which is of submillimeter accuracy. As a result, the accuracies of camera calibration and target positioning can meet the requirements of the accuracy of underwater 3D displacement measurement scenario.

Table 1: 3D coordinates discrepance of the check points between videogrammetric technique and total station

\begin{tabular}{|c|c|c|c|}
\hline \multirow{2}{*}{ No. } & \multicolumn{3}{|c|}{ Discrepance (mm) } \\
\cline { 2 - 4 } & $\Delta \mathrm{X}$ & $\Delta \mathrm{Y}$ & $\Delta \mathrm{Z}$ \\
\hline 1 & -0.38 & -0.21 & -0.07 \\
\hline 2 & -0.94 & 0.07 & -0.06 \\
\hline 3 & -0.85 & -0.08 & 0.11 \\
\hline 4 & 0.48 & -0.04 & 0.11 \\
\hline 5 & 0.35 & -0.05 & -0.17 \\
\hline 6 & -0.55 & 0.09 & -0.03 \\
\hline RMS & 0.63 & 0.10 & 0.10 \\
\hline
\end{tabular}

Through the aforementioned data processing, the 3D displacement of the structure could be obtained. As shown in Fig. 7, the 3D displacement curves are continuous and quite smooth. The periodically change in 3D displacement conforms to the influence of periodic wave on structure. The vibration of cameras caused by waves gives rise to the slight noises near peak most likely and this problem may be solved through filter processing algorithm such as Savitzky-Golay filter.

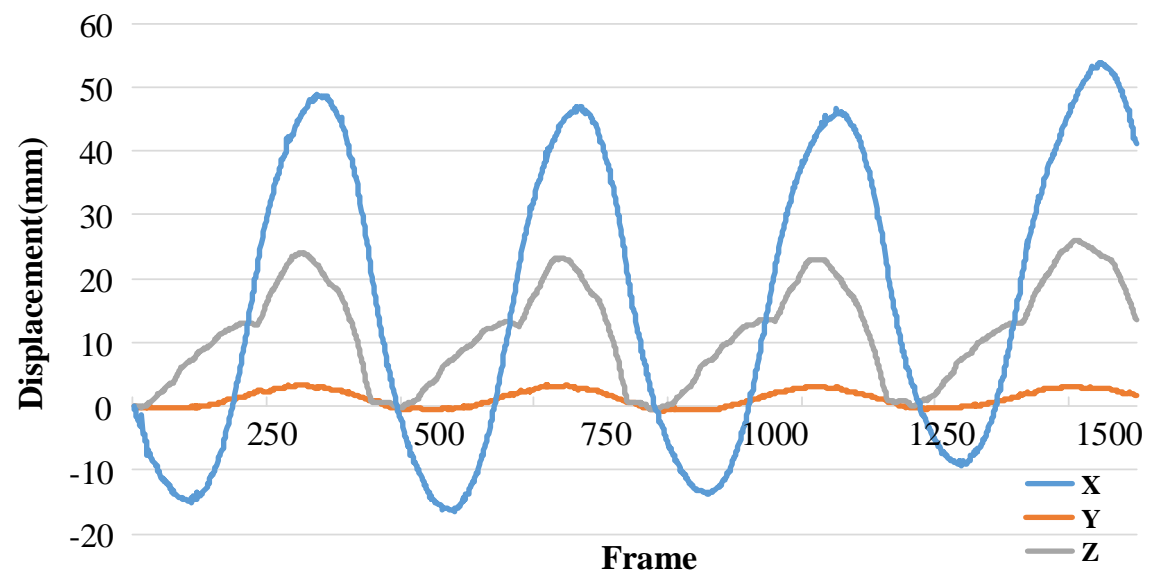

Fig. 7: 3D displacement measurement results of underwater structure.

\section{Conclusion}

This paper presents the application of high-speed videogrammetric technique in underwater 3D displacement measurement scenario. Through the underwater experiment, it is verified that the accuracy of underwater high-speed videogrammetric technique could reach submillimeter, which meets the accuracy requirement of underwater 3D displacement measurement. Moreover, the 3D displacement result indicates that high-speed videogrammetric technique is a feasible and reliable technique to measure the 3D displacement of underwater structures. In future, we will introduce this technique to more complex 
underwater scenario such as underwater shaking table test, for obtaining mechanical parameters such as transient deformation in the complex water environment.

\section{Acknowledgements}

This research was substantially supported by the National Key Research and Development of China (Project nos. 2016YFC0803107), the Natural Science Foundation of Shanghai National (Project nos. 20ZR1459800), and the State Key Laboratory of Disaster Reduction in Civil Engineering (SLDRCE19-B-37, SLDRCE19-A-12).

\section{References}

[1] Y. Wu, R. Nian, and B. He. 3D reconstruction model of underwater environment in stereo vision system. 2013 OCEANS-San Diego. IEEE. 2013, pp. 1-4.

[2] D. Rizzini, F. Kallasi, J. Aleotti, F. Oleari, and S. Caselli. Integration of a stereo vision system into an autonomous underwater vehicle for pipe manipulation tasks. Computers \& Electrical Engineering, 2017, 58: 560-571.

[3] M. Pankow, B. Justusson, and A. M. Waas. Three-dimensional digital image correlation technique using single high-speed camera for measuring large out-of-plane displacements at high framing rates. Applied optics, 2010, 49 (17): 3418-3427.

[4] M. Sutton, X. Ke, S. Lessner, M. Goldbach, M. Yost, F. Zhao, and H. Schreier. Strain field measurements on mouse carotid arteries using microscopic three-dimensional digital image correlation. Journal of Biomedical Materials Research Part A, 2008, 86 (2): 178-190.

[5] R. Severn. The development of shaking tables-a historical note. Earthquake engineering \& structural dynamics, 2011, 40 (2): 195-213.

[6] C. Pang, G. Y. Lee, T. Kim, S. Kim, H. Kim, S. Ahn, and K. Suh. A flexible and highly sensitive strain-gauge sensor using reversible interlocking of nanofibres. Nature Materials, 2012, 11(9): 795-801.

[7] X. Liu, X. Tong, X. Yin, X. Gu, and Z. Ye. Videogrammetric technique for three-dimensional structural progressive collapse measurement. Measurement, 2015, 63: 87-99.

[8] D. Feng, M. Feng, E. Ozer, and Y. Fukuda. A vision-based sensor for noncontact structural displacement measurement. Sensors, 2015, 15(7): 16557-16575.

[9] J. Fryer, and C. Fraser. On the calibration of underwater cameras. The Photogrammetric Record, 1986, 12(67): 7385.

[10] A. Fitzgibbon, M. Pilu, and R. Fisher. Direct least square fitting of ellipses. IEEE Transactions on pattern analysis and machine intelligence, 1999, 21(5): 476-480.

[11] B. Pan. Recent progress in digital image correlation. Experimental mechanics, 2011, 51(7): 1223-1235.

[12] F. Ackermann. Digital image correlation: performance and potential application in photogrammetry. The Photogrammetric Record, 1984, 11(64): 429-439. 